Journal of Advanced Research in Fluid Mechanics and Thermal Sciences

Journal homepage: www.akademiabaru.com/arfmts.html ISSN: $2289-7879$

\title{
Quasi Three-Dimensional Design for a Novel Turbo-Vapor Compressor and the Last Stage of a Low-Pressure Steam Turbine
}

\author{
Amin Mobarak ${ }^{1,}{ }^{*}$, Mostafa Shawky Abdel Moez ${ }^{1}$, Shady Ali $^{1}$ \\ 1 Mechanical Power Engineering Department, Faculty of Engineering, Cairo University, Egypt
}

\begin{tabular}{|c|c|}
\hline ARTICLE INFO & ABSTRACT \\
\hline $\begin{array}{l}\text { Keywords: } \\
\text { Freshwater and electricity production; } \\
\text { turbo-vapor compressor; low-pressure } \\
\text { steam turbine }\end{array}$ & $\begin{array}{l}\text { Turbo-vapor compressors (TVCS) are used to create a vacuum pressure in the } \\
\text { evaporator of a novel combined cycle for electricity and freshwater production were } \\
\text { invented. A novel design conceived of a TVC is introduced to increase the efficiency, } \\
\text { allowable mass flow rate and reduce costs and losses. The system consists of a single } \\
\text { axial compressor rotor followed by a single axial turbine rotor which drives the } \\
\text { upstream compressor, allowing high flow rates. A quasi-3D design is carried out for the } \\
\text { TVC to calculate the flow velocity components and angles and ensure that the turbo- } \\
\text { vapor turbine work is equal to the turbo-vapor compressor work. A preliminary design } \\
\text { of the low-pressure power turbine (LPT) is done to examine the size and number of } \\
\text { stages. The (LPT) size is twice the size of TVC at typical cycle operating conditions. A } \\
\text { three-stage design is the most appropriate choice for the number of stages. It satisfies } \\
\text { the accelerating relative flow condition at the last stage over a range of flow } \\
\text { coefficients. A quasi-3D design is carried out for the LPT's last stage to ensure a multi- } \\
\text { stage power turbine's safe design. }\end{array}$ \\
\hline
\end{tabular}

\section{Introduction}

There is a significant need for green electricity production due to crude oil and natural gas depletion all over the world, especially Europe [1] and some researches on electricity consumption optimization [2]. There is an immediate need for freshwater production due to water shortages in some regions, such as deserts. Several authors developed methods to produce freshwater from saltwater using solar energy [3, 4]. In contrast, other authors developed new methods for water consumption in agriculture [5]. A novel combined electricity and freshwater production thermal cycle developed by Amin Mobarak [6, 7] can produce a massive amount of fresh water and electricity at a higher efficiency. The novel cycle operates at low temperatures $\left(60-90^{\circ} \mathrm{C}\right)$ using water vapor as a working medium under pressure below the atmospheric pressure. This is achieved by a turbo-vapor compressor, TVC (to create negative pressure in the evaporator), a conventional low-pressure water vapor turbine, LPT (to produce electricity), and a condenser working in an open cycle. The current

\footnotetext{
* Corresponding author.

E-mail address: amobarak@alwatania-egy.com
}

https://doi.org/10.37934/arfmts.85.2.113 
work focuses on the quasi 3D design of the turbo-vapor compressor and some preliminary design aspects concerning the low-pressure water vapor turbine.

In this research, a parametric study between the last stage of low-pressure water vapor turbine and turbo-vapor compressor is done to examine the effect of evaporator temperature, rotational speed, and hub flow coefficient ratio $\phi_{h, T V C} / \phi_{h, L P T}$ on the size or diameter ratio $D_{t, L P T} / D_{t, T V C}$. Moreover, the authors proposed a novel TVC design in Patent No. 2020/798 [8] (under review) using a quasi three-dimensional design to obtain the velocity triangles at different spanwise sections. The water vapor properties are estimated at the inlet and exit from the turbo-vapor compressor and at the turbo-vapor turbine's exit. Finally, a preliminary design of the low-pressure water vapor turbine is carried out to minimize the stages. The last stage of the LPT is designed based on the same methodology used in the TVC to ensure that the entire turbine operates efficiently over a wide range of flow coefficients.

\section{Selection of Turbo-Vapor Compressor}

\subsection{Selection of Type and Blade Row of the TVC}

The turbo-vapor compressor consists of a water vapor turbine that drives the water vapor compressor to create a suction pressure at the compressor inlet for industrial purposes. The first suggested design can be a centrifugal compressor followed by a centrifugal turbine (e.g., Turbocharger) $[9,10]$, as shown in Figure 1 . The centrifugal compressor consists of an impeller, diffuser ring, and scroll casing. The centrifugal turbine consists of a volute casing, nozzle ring, rotor, and a diffuser. The second suggested design can be an axial compressor stage equipped with a rotor, diffuser, and possibly, inlet guide vanes. The compressor is driven by an axial turbine stage, which comprises a stator, rotor, and possibly, outlet guide vanes.

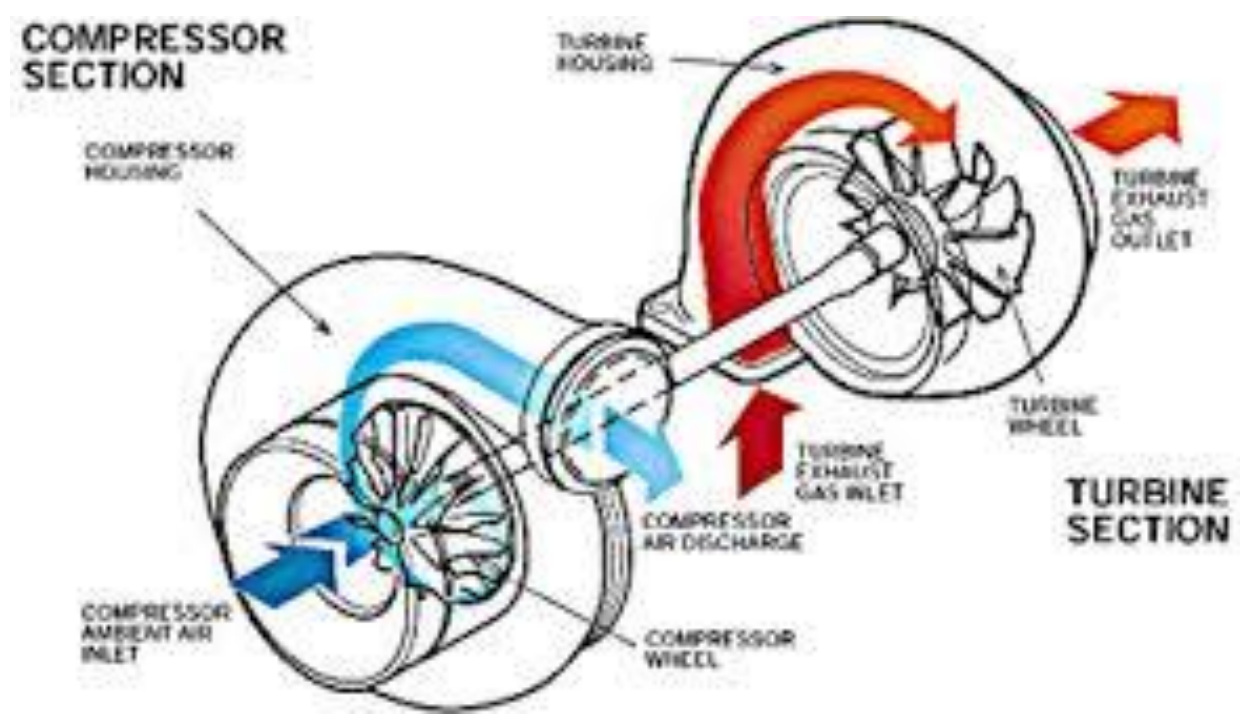

Fig. 1. Turbocharger [9]

In the present work, a novel design of a turbo-vapor compressor consists of an axial compressor rotor driven by an axial turbine rotor without any stators for the turbine or compressor to avoid extra losses, as shown in Figure 2. The authors propose the design in Patent No. 2020/798 [8]. A starting electric motor is used to start the compressor and turbine by using a clutch coupling between the staring motor and turbo-vapor compressor shafts. After the system reaches a steady state (turbine work equals compressor work), the clutch is decoupled to allow the turbine to run the compressor. 
The compressor and turbine are using the same shaft, which means they run the same speed. Fortunately, the current application requires a small pressure ratio of the order of 1.05. Therefore, a single axial compressor rotor driven by a single axial turbine rotor is the most economical design. Axial compressor design can accommodate more airflow than a centrifugal design of the same size but provides lower pressure ratios. The Turbo-vapor compressor is selected as an axial flow compressor to accommodate the water vapor mass flow rate of $100 \mathrm{~kg} / \mathrm{sec}$.

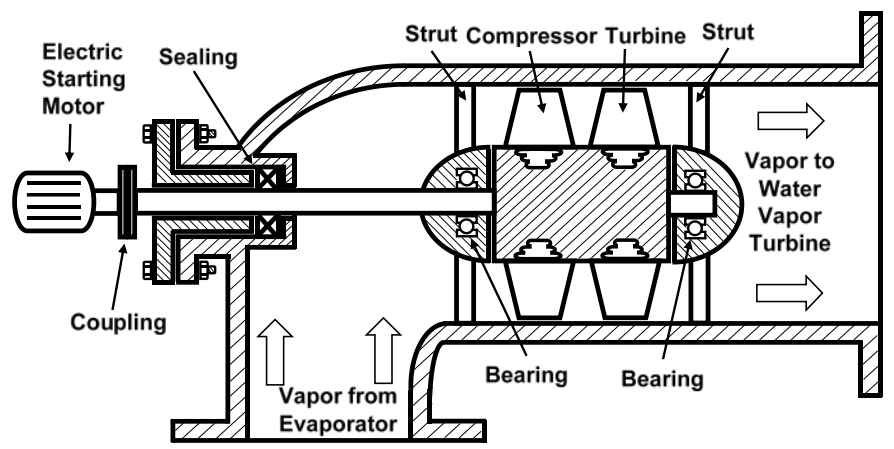

Fig. 2. Turbo-vapor compressor layout [8]

\subsection{Selection of LPT to TVC Diameter Ratio}

In this Section, the size limits and ratios of the LPT and TVC are examined. Some parameters are tested to determine the size ratio of the LPT to TVC. It is found that the design ratio $D_{t, L P T} / D_{t, T V C}$ should be kept around 2.0 for safe operation [11]. By applying the continuity equation along with the TVC and the LPT in addition to some manipulations, Eq. (1) [12] yields

$$
\begin{aligned}
& \rho_{T V C} \frac{\pi}{4} D_{t, T V C}^{3}\left(1-v_{T V C}^{2}\right) v_{T V C} \frac{\pi N_{T V C}}{60} \phi_{h, T V C}= \\
& \rho_{L P T} \frac{\pi}{4} D_{t, L P T}^{3}\left(1-v_{L P T}^{2}\right) v_{L P T} \frac{\pi N_{L P T}}{60} \phi_{h, L P T}
\end{aligned}
$$

where the axial hub velocity $C a_{h}$ is kept constant along with the TVC and LPT. The hub-to-tip diameter ratios $v_{T V C} v_{L P T}$ are set to be 0.5 to account for the worst-case scenario in terms of blade twist and reaction. The hub section is selected as it is the most critical Section in terms of flow deflection. Eq. (1) is used for the following analysis to choose the design diameter ratio $D_{t, L P T} / D_{t, T V C}$ based on some input parameters.

\subsubsection{Effect of evaporator temperature}

The evaporator temperature on the diameter ratio $D_{t, L P T} / D_{t, T V C}$ is investigated when $N \phi_{h} v$ held constant for both LPT and TVC. The condenser's exit temperature is roughly constant at $30^{\circ} \mathrm{C}$, so the density at the exit from the stage of multi-stage low-pressure water vapor turbine is assumed to be similar to that of the condenser $\left(0.0304 \mathrm{~kg} / \mathrm{m}^{3}\right)$. Changing the evaporator temperature from $70^{\circ}$ to $90^{\circ} \mathrm{C}$ will increase $D_{t, L P T} / D_{t, T V C}$ from 1.8 to 2.4 , as shown in Figure 3. 


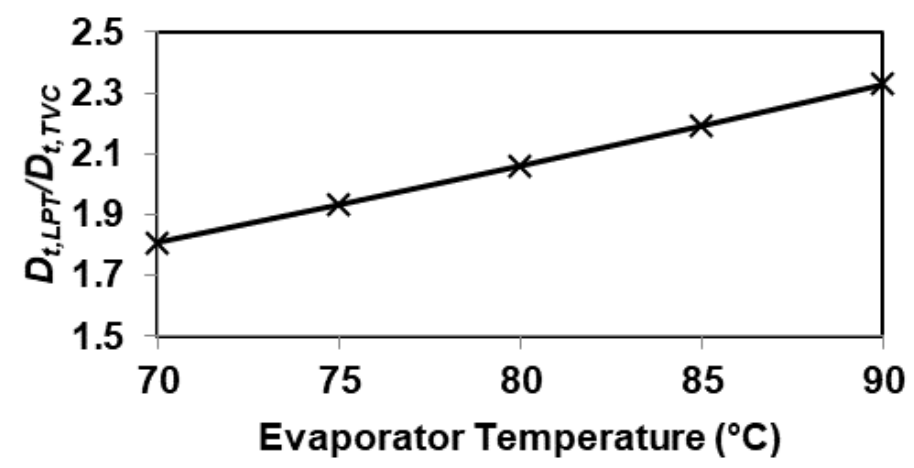

Fig. 3. Effect of evaporator temperature on $D_{t, L P T} / D_{t, T V C}$

\subsubsection{Effect of TVC rotational speed}

The effect of $N_{T V C}$ on $D_{t, L P T} / D_{t, T V C}$ is investigated when evaporator temperature $\phi_{h} v$ and is held constant for both LPT and TVC. The low-pressure water vapor turbine's rotational speed is also kept constant at $3000 \mathrm{rpm}$ [11]. The turbo-vapor compressor's rotational speed changes from 1500 to $4500 \mathrm{rpm}$ - consequently, the diameter ratio $D_{t, L P T} / D_{t, T V C}$ increases from 1.7 to 2.4 , as shown in Figure 4.

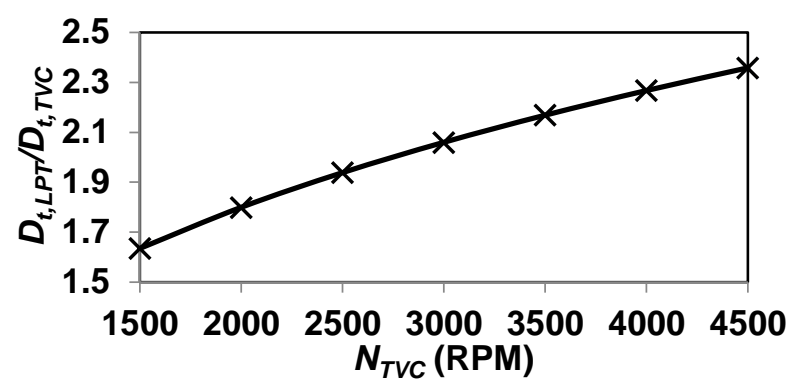

Fig. 4. Effect of $N_{T V C}$ on $D_{t, L P T} / D_{t, T V C}$

\subsubsection{Effect of hub flow coefficient ratio}

The effect of $\phi_{h, T V C} / \phi_{h, L P T}$ on $D_{t, L P T} / D_{t, T V C}$ is investigated when $N$ and $v$ for both LPT and TVC, and the evaporator temperature is held constant as the ratio $\phi_{h, T V C} / \phi_{h, L P T}$ changes from 0.7 to 1.3. The diameter ratio $D_{t, L P T} / D_{t, T V C}$ increases from 1.8 to 2.2, as shown in Figure 5.

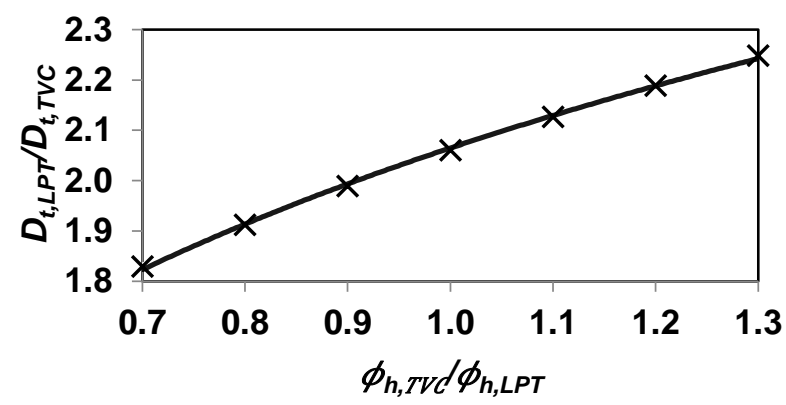

Fig. 5. Effect of $\phi_{h, T V C} / \phi_{h, L P T}$ on $D_{t, L P T} / D_{t, T V C}$ 
It is evident from the previous analysis that the LPT diameter is around twice the TVC diameter for an evaporator temperature of $80^{\circ} \mathrm{C}$, a condenser temperature of $30^{\circ} \mathrm{C}, N_{T V C}=N_{L P T}=3000 \mathrm{rpm}$ and $\phi_{h, T V C} / \phi_{h, L P T}=1$.

\section{Calculations of TVC Tip and Hub Diameters}

The sizing of the tip diameter of the TVC is carried out based on a maximum flow rate of 100 $\mathrm{kg} / \mathrm{sec}$, hub-to-tip diameter ratio ranging from $v=0.5$ to $v=0.8$, and unity $\phi_{h}$ [13]. By applying the continuity equation at the inlet of the TVC as well as performing some mathematical manipulations, Eq. (2) yields

$$
D_{t}^{3}=\frac{240 \times v_{T V C 1} \times \dot{m}_{T V C}}{\pi^{2} \times v\left(1-v^{2}\right) \times \phi_{h} \times N}
$$

where the inlet specific volume $v_{T V C 1}$ is the saturated vapor specific volume at $80^{\circ} \mathrm{C}$. The absolute inlet velocity is axial (no inlet guide vanes) and is assumed to be constant along the spanwise direction. The rotational speed $N$ is held constant at $3000 \mathrm{rpm}$. MATLAB R2018a and the function XSteam [14] are used for mathematical calculations and vapor properties calculations.

The first tip diameter is calculated based on a flow rate of $100 \mathrm{~kg} / \mathrm{s}$ and $v=0.5$ from Eq. (2). Afterward, the tip diameter is fixed, and the iteration is applied on Eq. (2) to find the ratio $v$ for mass flow rates below $100 \mathrm{~kg} / \mathrm{s}$ with $10 \mathrm{~kg} / \mathrm{s}$ step. The flow area reduction associated with flow rate reduction leads to an increase in the hub diameter $v$. For a mass flow of $70 \mathrm{~kg} / \mathrm{s}$, the ratio $v$ reaches 0.83 (at $D_{t}=1.9452 \mathrm{~m}$ ), exceeding the upper limit set for that ratio. Therefore, the ratio $v$ is set back to 0.5 at $70 \mathrm{~kg} / \mathrm{s}$. New tip diameter is calculated, and iterations are performed to obtain new ratios $v$ at flow rates below $70 \mathrm{~kg} / \mathrm{s}$ while setting the upper limit of 0.8 . The procedure is repeated until a mass flow of $10 \mathrm{~kg} / \mathrm{s}$ is reached, as shown in Table 1 . In this study, the mass flow rate of $50 \mathrm{~kg} / \mathrm{s}$ and a hub-to-tip ratio, $v=0.5$, are applied for the TVC design. The static inlet conditions are assumed to be saturated vapor at the evaporator temperature of $80^{\circ} \mathrm{C}$.

Table 1

\begin{tabular}{llll}
\multicolumn{4}{c}{ Summary of TVC sizing results } \\
\hline$\dot{m}_{T V C}(\mathrm{~kg} / \mathrm{s})$ & $D_{t}(\mathrm{~m})$ & $D_{h}(\mathrm{~m})$ & $v$ \\
\hline 10 & 0.9029 & 0.4514 & 0.5000 \\
20 & 1.1376 & 0.5688 & 0.5000 \\
30 & 1.3022 & 0.6511 & 0.5000 \\
40 & 1.5439 & 1.2143 & 0.7865 \\
50 & 1.5439 & 0.7720 & 0.5000 \\
60 & 1.7272 & 1.3117 & 0.7594 \\
70 & 1.7272 & 0.8636 & 0.5000 \\
80 & 1.9452 & 1.5299 & 0.7865 \\
90 & 1.9452 & 1.4311 & 0.7357 \\
100 & 1.9452 & 0.9726 & 0.5000 \\
\hline
\end{tabular}

\section{Velocity Triangles and Shape of blades for the TVC}

The hub-to-tip diameter ratio of 0.5 is selected since it is associated with high losses, circulation, and boundary layer problems. If the design is acceptable in this case, it will be adequate for hub-totip diameter ratios [13]. Therefore, a mass flow rate of $50 \mathrm{~kg} / \mathrm{s}$ ensures that the turbo-vapor 
compressor operates safely in the most critical conditions. The deceleration rate should be maintained above the limit, $W_{2} / W_{1} \geq 0.72$, to avoid separation [15]. Flow separation occurs when the boundary layer travels far enough against an adverse pressure gradient. The speed of the boundary layer relative to the wall falls almost to zero. The fluid flow becomes detached from the object's surface and takes the forms of eddies and vortices. In aerodynamics, flow separation leads to increased drag, particularly pressure drag, caused by the pressure differential between the object's front and rear surfaces as it travels through the air.

\subsection{One-Dimensional Design at the Hub Section}

The primary compressor inlet and exit velocity triangles are shown in Figure 6 . The axial velocity is assumed to be constant, along with spanwise and streamwise directions. The flow angles are measured from the axial direction. For a mass flow rate of $50 \mathrm{~kg} / \mathrm{s}$, inlet temperature $=80^{\circ} \mathrm{C}, N$ $=3000 \mathrm{rpm}, \phi_{h}=1, D_{t}=1.5439 \mathrm{~m}$ and $v=0.5$, velocity components and angles are readily calculated at the inlet. A deceleration ratio is set as 0.85 at the hub to ensure safe diffusion at all spanwise sections. Using the given deceleration ratio and the assumption of constant streamwise axial velocity, all velocity components and angles are calculated at the hub outlet. The stagger angle is assumed to be the arithmetic average of the relative flow angles at the compressor's inlet and outlet. Table 2 (Hub Section column) shows the calculation results in the hub section. The compressor's specific work at the hub is $4895 \mathrm{~J} / \mathrm{kg}$, which is assumed to be constant along the span. The hub flow turning, which is the maximum turning along the span, is $11.3^{\circ}$. This means that there is no risk of boundary layer separation at the suction surface of compressor blades. Figure 8 (a) shows the velocity triangles and shape of blades at the compressor inlet at the hub (first two triangles from left).

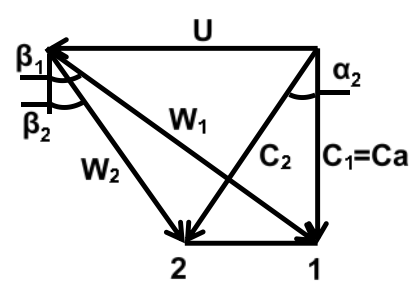

Fig. 6. Basic compressor velocity triangle

Table 2

Calculated compressor velocities and angles at three different sections

\begin{tabular}{|c|c|c|c|}
\hline & Hub section & Mean section & Tip section \\
\hline$D(m)$ & 0.7720 & 1.1579 & 1.5439 \\
\hline$U(\mathrm{~m} / \mathrm{s})$ & 121.2597 & 181.8896 & 242.5194 \\
\hline$C_{1}=C a(m / s)$ & & 121.2597 & \\
\hline$W_{1}(\mathrm{~m} / \mathrm{s})$ & 171.4871 & 218.6041 & 271.1450 \\
\hline$B_{1}$ (degree) & 45.000 & 56.3099 & 63.4349 \\
\hline$W_{2}(\mathrm{~m} / \mathrm{s})$ & 145.7641 & 196.7782 & 253.2521 \\
\hline$W u_{2}(\mathrm{~m} / \mathrm{s})$ & 80.8903 & 154.9766 & 222.3347 \\
\hline $\mathrm{Cu}_{2}(\mathrm{~m} / \mathrm{s})$ & 40.3694 & 26.9129 & 20.1847 \\
\hline $\mathrm{B}_{2}$ (degree) & 33.7066 & 51.9590 & 61.3923 \\
\hline Work (J/kg) & & 4895.1811 & \\
\hline Stagger, $v$ (degree) & 39.3533 & 54.1345 & 62.4136 \\
\hline Turning, $\vartheta$ (degree) & 11.2934 & 4.3509 & 2.0427 \\
\hline
\end{tabular}


4.2 Quasi Three-Dimensional Design at the Tip and Mean Sections (Based on Simple Free Vortex Design)

Assuming free vortex design, work and axial velocity are constant along span [13]. The inlet velocity diagram can be calculated at any spanwise section given that inlet axial velocity, which is constant, and blade speed are known at any section. The exit velocity diagram can be obtained at any section, given that the spanwise work and axial velocity are constant at all sections. Table 2 (Mean and Tip Sections) lists the velocity components and angles at the mean and tip sections. Again, the stagger is assumed to be the arithmetic average of the inlet and outlet relative flow angles. Figure 7 (Left) shows the compressor blade's preliminary stacked profiles at the three main sections based on the NACA65 series profile. The velocity triangles along with the shape of blades at mean and tip sections are shown in the first and second diagrams of Figure 8 (b, c)

\section{Calculations for Turbo-Vapor Turbine}

The Turbo-vapor turbine has the same hub, mean and tip diameters calculated from the compressor at $50 \mathrm{~kg} / \mathrm{s}$ and the same rotational speed. The specific work of the turbo-vapor turbine is assumed to be equal to that of the turbo-vapor compressor. The axial flow velocity is considered to be constant along the streamwise and spanwise direction. The turbine-specific work is also constant along the spanwise constant based on free vortex design. It is noted that the stagger and turning angles are identical to those of the compressor due to the assumption of constant work and axial velocity. The turbine exit velocities and angles are similar to those of the compressor inlet, as shown in Table 3. Figure 7 (right) shows the stacked turbine profiles at the main three sections. Figure 8 (third column of diagrams) shows the turbine exit velocity diagrams. The absolute exit velocity is axial to avoid the installation of outlet guide vanes.

Table 3

Calculated TVC turbine velocities and angles at three different sections

\begin{tabular}{llll}
\hline & Hub section & Mean section & Tip section \\
\hline$D(\mathrm{~m})$ & 0.7720 & 1.1579 & 1.5439 \\
$U(\mathrm{~m} / \mathrm{s})$ & 121.2597 & 181.8896 & 242.5194 \\
$C_{3}=C a(\mathrm{~m} / \mathrm{s})$ & & 121.2597 & \\
$W_{3}(\mathrm{~m} / \mathrm{s})$ & 171.4871 & 218.6041 & 271.1450 \\
$B_{3}($ degree $)$ & 45.000 & 56.3099 & 63.4349 \\
Work $(\mathrm{J} / \mathrm{kg})$ & & 4895.1811 & \\
Stagger, $\gamma$ (degree) & 39.3533 & 54.1345 & 62.4136 \\
Turning, $\vartheta$ (degree) & 11.2934 & 4.3509 & 2.0427 \\
\hline
\end{tabular}

\section{Calculations of Water Vapor Properties Across Turbo-Vapor Compressor}

At the inlet to the axial compressor, the static temperature of water vapor is $80^{\circ} \mathrm{C}$. The static inlet conditions are assumed to be saturated vapor. To find the outlet conditions, the compressor and turbine total-to-total efficiencies are considered to be 0.92 and 0.94 , respectively. The water vapor properties are calculated using the MATLAB function XSteam [14]. Figure 9 shows the calculations flow chart. Table 4 shows the output values of the water vapor properties across the TVC. 


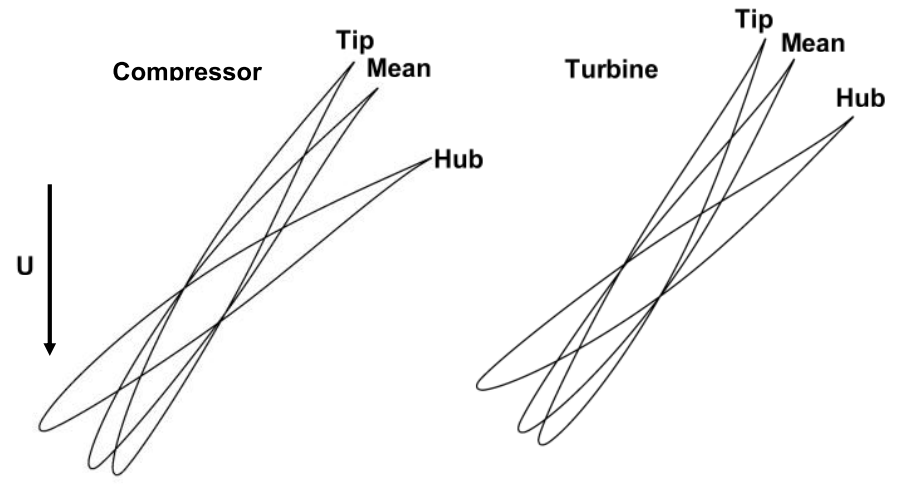

Fig. 7. Stacked profiles at hub, mean, and tip of axial turbo-vapor compressor and turbine

(a)

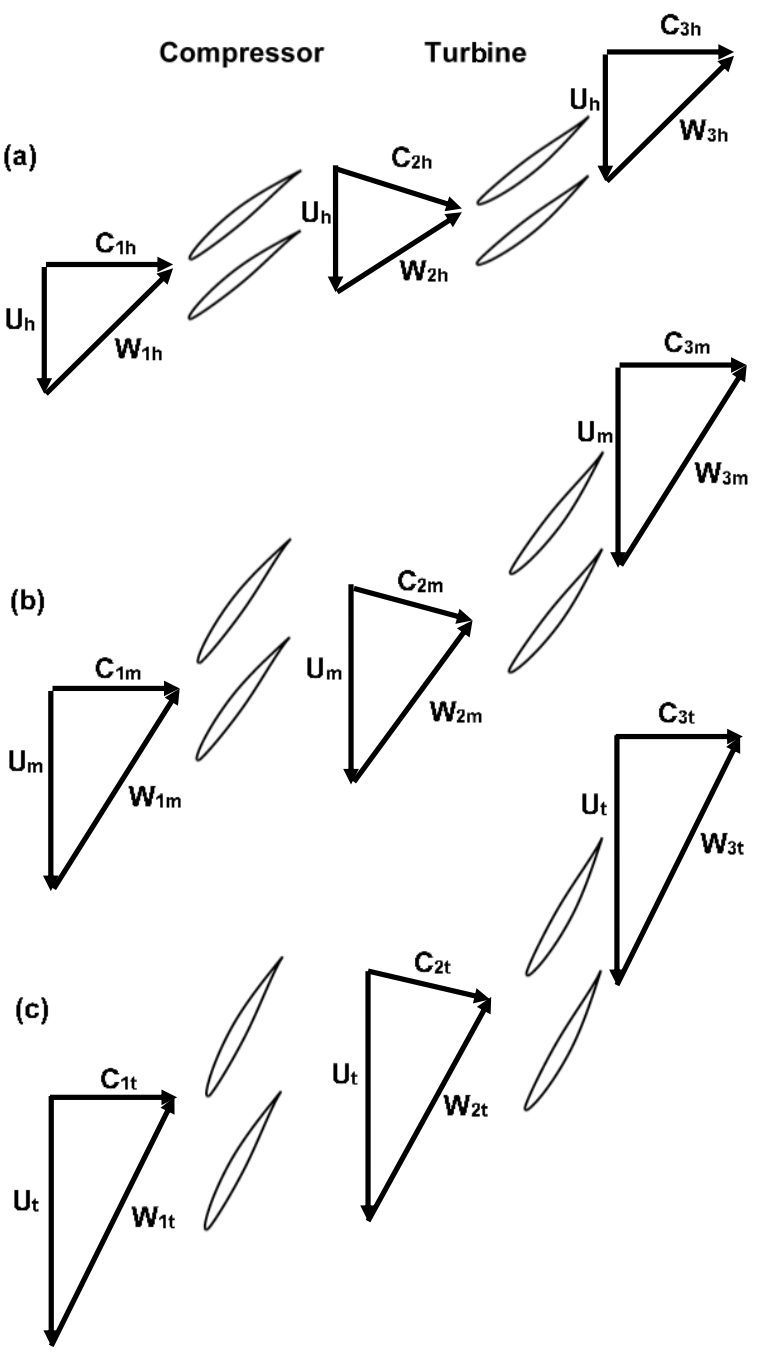

Fig. 8. Velocity triangles and shape of blades at (a) Hub section, (b) Mean section, and (c) Tip section across the axial turbo-vapor

$$
\begin{aligned}
& t_{1}=t_{\text {Sat.Vap. }} \rightarrow p_{1}, h_{1}, s_{o 1}, v_{1} \\
& C_{1}=C a, h_{1} \rightarrow h_{o 1} \\
& h_{o 1}, s_{o 1} \rightarrow p_{o 1}, t_{o 1} \\
& \text { work, } h_{o 1} \rightarrow h_{o 2} \\
& \boldsymbol{h}_{o 1}, \boldsymbol{h}_{o 2}, \eta_{t t, C} \rightarrow \boldsymbol{h}_{o 2 s} \\
& h_{o 2 s}, s_{o 1} \rightarrow p_{o 2} \\
& p_{o 2}, h_{o 2} \rightarrow s_{o 2}, t_{o 2} \\
& h_{o 2}, C_{2} \rightarrow h_{2} \\
& h_{2}, s_{o 2} \rightarrow p_{2}, t_{2} \\
& p_{2}, s_{o 2} \rightarrow v_{2} \\
& \begin{array}{c}
\hline \multicolumn{1}{c}{\rightarrow h_{o 1}} \\
h_{o 2}, h_{o 3}, \eta_{t t, T} \rightarrow h_{o 3 s} \\
h_{o 3 s}, s_{o 2} \rightarrow p_{o 3} \\
p_{o 3}, h_{o 3} \rightarrow s_{o 3}, t_{o 3} \\
h_{o 3}, C_{3} \rightarrow h_{3} \\
h_{3}, s_{o 3} \rightarrow p_{3}, t_{3} \\
p_{3}, s_{o 3} \rightarrow v_{3}
\end{array}
\end{aligned}
$$

Fig. 9. Procedures of properties calculations across the TVC 
Table 4

Water vapor properties across turbo-vapor compressor

\begin{tabular}{llll}
\hline Properties & Compressor Inlet & Compressor Exit & Turbine Exit \\
\hline$t_{o}\left({ }^{\circ} \mathrm{C}\right)$ & 83.8501 & 86.4043 & 83.8324 \\
$p_{o}(\mathrm{bar})$ & 0.4961 & 0.5099 & 0.4940 \\
$h_{o}(\mathrm{~kJ} / \mathrm{kg})$ & 2650.367 & 2655.2615 & 2650.37 \\
$s_{o}(\mathrm{~kJ} / \mathrm{kg} . \mathrm{K})$ & 7.6110 & 7.6121 & 7.6130 \\
$t\left({ }^{\circ} \mathrm{C}\right)$ & 80.0000 & 82.1277 & 79.9851 \\
$p(\mathrm{bar})$ & 0.4741 & 0.4851 & 0.4721 \\
$\mathrm{~h}(\mathrm{~kJ} / \mathrm{kg})$ & 2643.01 & 2647.0946 & 2643.01 \\
$U\left(\mathrm{~m}^{3} / \mathrm{kg}\right)$ & 3.4053 & 3.3412 & 3.4202 \\
\hline
\end{tabular}

\section{Multi-Stage Low-Pressure Water Vapor Turbine Design}

\subsection{Multi-Stage Low-Pressure Water Vapor Turbine Preliminary Calculations}

The inlet and exit conditions of the LPT should be known for the calculation procedures. The LPT's inlet conditions are assumed to be the same as the turbo-vapor turbine's exit conditions listed in Table 4. The LPT's exit pressure is considered to be related to the condenser saturated pressure at $30^{\circ} \mathrm{C}$ through the static pressure recovery coefficient. A diverging duct is placed between the LPT exit and the condenser inlet. The LPT's total-to-total efficiency is assumed to be 0.9 [16], and the pressure recovery coefficient is considered to be 0.12 [17]. The pressure recovery coefficient is given by Eq. (3)

$$
C_{p}=\frac{p_{\text {cond }}-p_{4}}{0.5\left(\rho C a^{2}\right)_{4}}
$$

$p_{\text {cond }}$ The saturation pressure at $30^{\circ} \mathrm{C}$ and subscript 4 represent the conditions at the low-pressure turbine's exit. The LPT is designed at $3000 \mathrm{rpm}, 50 \mathrm{~kg} / \mathrm{s}$ (same as TVC), and a range of flow coefficient, $\phi_{h}=0.8$ to $\phi_{h}=1.2$. Again, the axial velocity is assumed to be constant along the spanwise direction. The hub-to-tip diameter ratio $v$ is assumed to be 0.5 . Since the exit density or specific volume is not explicitly defined, an exit density and total-to-static efficiency are assumed. An iterative process is applied until the solution converges to the correct specific volume and targets total-to-total efficiency, as shown in Figure 10.

It should be noted that subscript 3 denotes the LPT inlet condition, which is identical to the TVC exit condition and subscript 4 represents the LPT exit condition. Table 5 lists some of the exit conditions at different flow coefficients. The total-to-static efficiency is lower at higher flow coefficients since it is associated with higher exit kinetic energy loss.

\subsection{Preliminary Design of the Number of Stages and The Last Stage of the LPT}

It is vital to determine the number of stages and the design of the last stage of turbines for sizing and economic reasons. The most economical choice is, of course, a single-stage design. However, a check should be performed on the acceleration ratio of the relative velocity across the turbine blades to ensure $W_{2}>W_{1}$. A series of trials to check the effect of the number of stages are performed, but the details are not listed in this paper.

The inlet and exit of the last stage will be denoted by 1 and 2, respectively. The inlet velocity diagram is calculated from work and assuming a constant streamwise axial velocity. The blade speed is already known in all sections from the procedures listed in Figure 10. The absolute exit velocity is 
considered to be purely axial. The inlet and exit velocity diagrams are fully defined. The hub section is the Section of interest since it shows the worst acceleration ratios and highest flow turning.

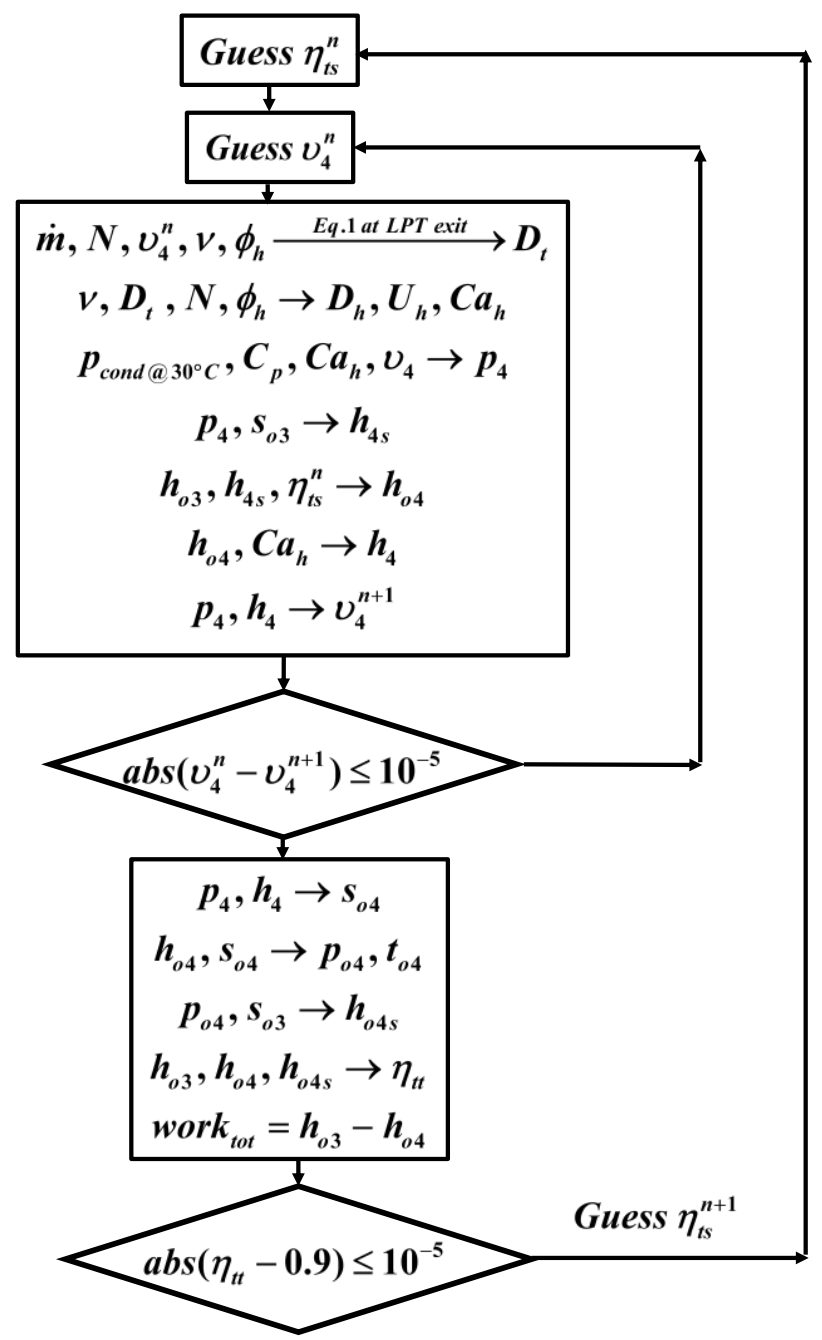

Fig. 10. Procedures of properties calculations across the LPT

The single-stage design gives $W_{2 h}<W_{1 h}$, which is inappropriate for turbines. For multi-stage designs, an equal specific work per stage is assumed. The two-stage design also gives decelerating relative flow $\left(W_{2 h}<W_{1 h}\right)$ at all flow coefficients, which should be avoided. A three-stage design gives $W_{2 h}>W_{1 h}$ at all flow coefficients. This is the most reliable and economical choice for the given conditions. Table 6 lists the acceleration ratio and turns for the LPT's last stage based on a threestage design. Figure 11 shows the velocity diagram at the hub, mean, and tip sections based on the free vortex design at $\phi_{h}=1.2$. The procedures of calculating the velocity diagrams for the LPT are the same as those for the TVC. Figure 12 depicts the LPT expansion process and a close-up of the TVC compression and expansion processes on the h-s diagram. Figure 13 shows a schematic drawing of the three-stage LPT and the upstream TVC, and the starting motor. All components utilize the same shaft and hence, the same rpm. 


\section{Table 5}

Multi-stage low-pressure turbine calculations results for three-stage design

\begin{tabular}{llll}
\hline$\phi_{h}$ & 0.8 & 1.0 & 1.2 \\
$D_{h}(\mathrm{~m})$ & 1.7266 & 1.6064 & 1.5152 \\
$D_{t}(\mathrm{~m})$ & 3.4532 & 3.2127 & 3.0304 \\
$U_{4}\left(\mathrm{~m}^{3} / \mathrm{kg}\right)$ & 30.4809 & 30.6819 & 30.8990 \\
$P_{o 4}(\mathrm{bar})$ & 0.0499 & 0.0529 & 0.0562 \\
$\mathrm{Cah}(\mathrm{m} / \mathrm{s})$ & 216.9713 & 252.3250 & 285.6069 \\
Total Work $(\mathrm{kJ} / \mathrm{kg})$ & 295.8470 & 289.3446 & 282.3160 \\
$\eta_{t s, L P T} \%$ & 84.0691 & 81.9978 & 79.7734 \\
\hline
\end{tabular}

Table 6

Calculations of the last stage of the multi-stage low-pressure turbine for three-stage design

\begin{tabular}{llll}
\hline$\phi_{h}$ & 0.8 & 1.0 & 1.2 \\
Stage Work $(\mathrm{kJ} / \mathrm{kg})$ & 98.6157 & 96.4482 & 94.1053 \\
$\alpha_{1}$ & 59.1747 & 56.5702 & 54.1579 \\
$W_{1 h}(\mathrm{~m} / \mathrm{s})$ & 235.8245 & 283.8050 & 326.1003 \\
$W_{2 h}(\mathrm{~m} / \mathrm{s})$ & 347.3235 & 356.8415 & 371.7769 \\
$W_{2 h} / W_{1 h}$ & 1.4728 & 1.2573 & 1.1401 \\
Turning, $\vartheta_{h}$ (degree) & 74.4062 & 72.2423 & 68.6628 \\
\hline
\end{tabular}
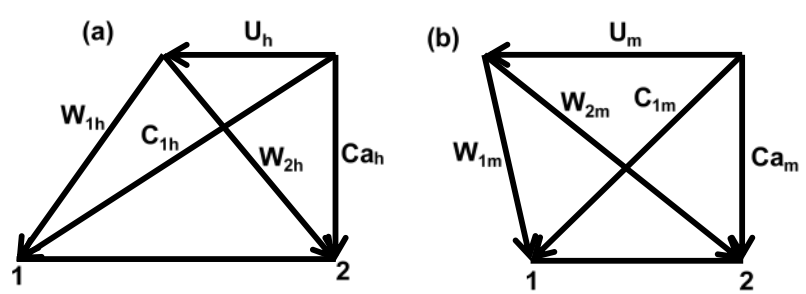

(c)

$\mathbf{U}_{\mathrm{t}}$

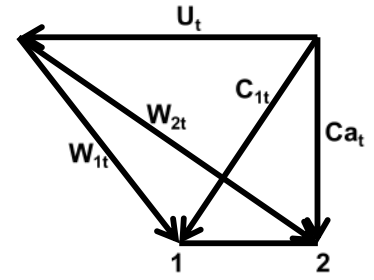

Fig. 11. Velocity triangles of the last stage of the lowpressure water vapor turbine at $\phi_{h}=1.2$ for threestage design at (a) Hub, (b) Mean, and (c) Tip sections

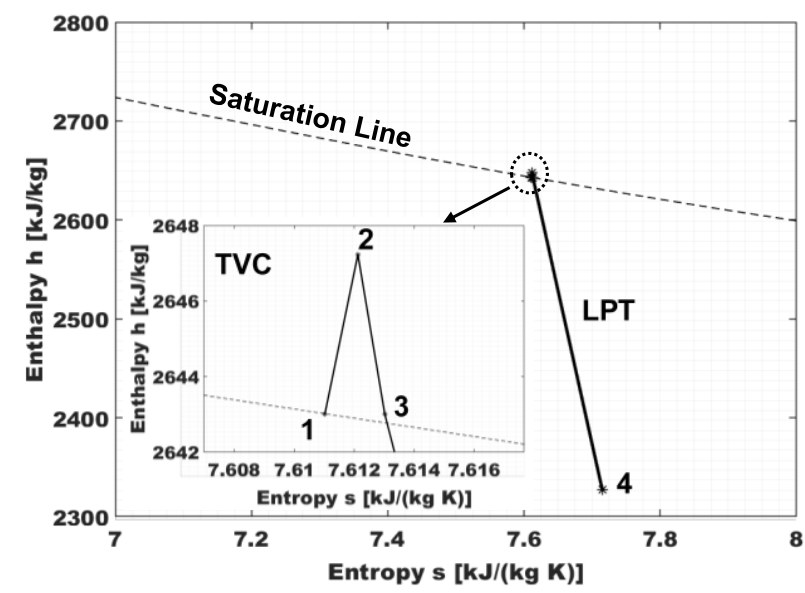

Fig. 12. Enthalpy-entropy diagram for the processes of the TVC and LPT 


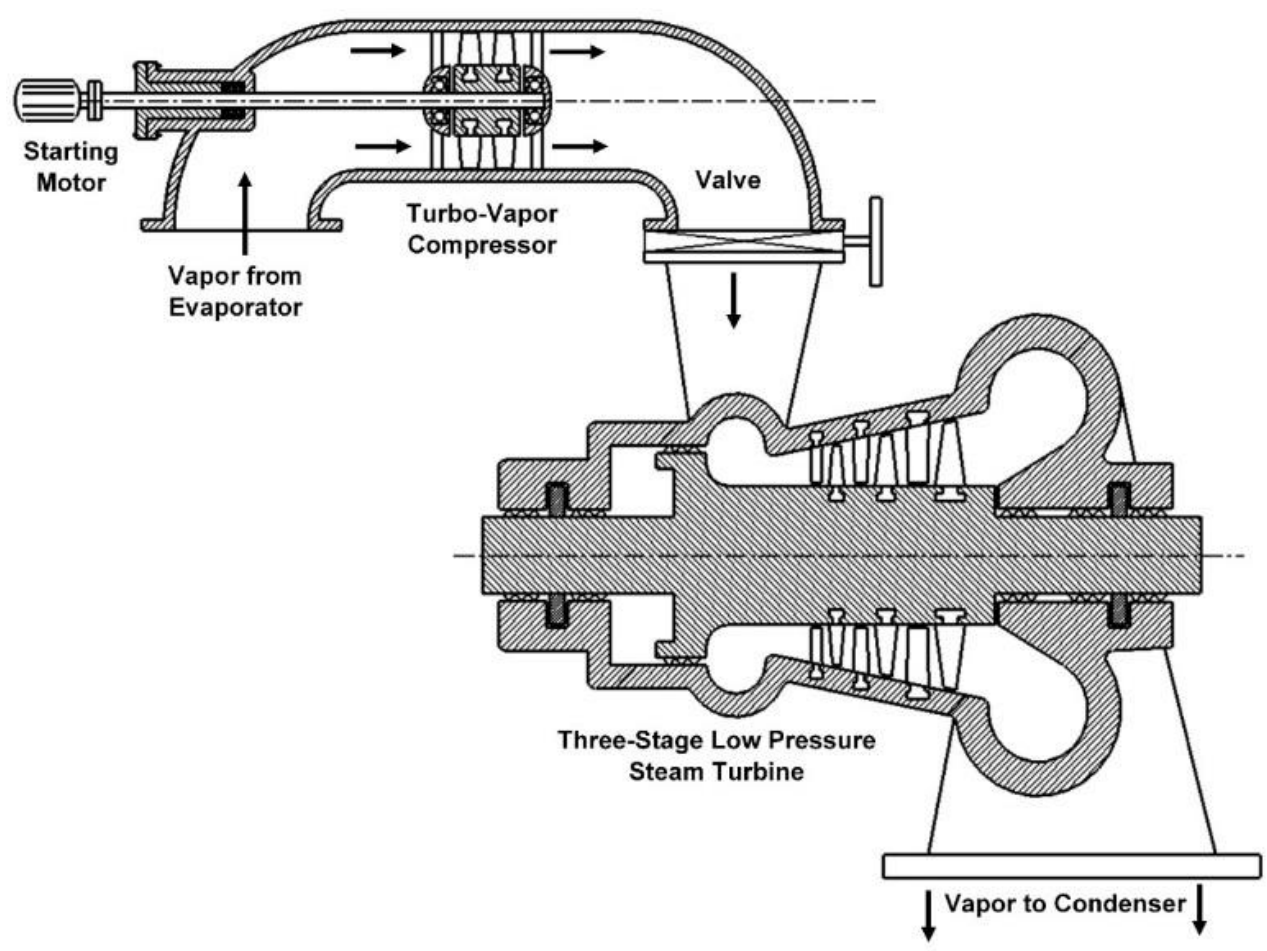

Fig. 13. A schematic drawing of the TVC and LPT

\section{Conclusions}

A preliminary design for some components of a novel freshwater and electricity production thermal cycle was carried out. This paper discussed the design of a novel TVC and a preliminary design of the low-pressure steam turbine. For typical operating conditions (evaporator temperature of $80^{\circ} \mathrm{C}$, $3000 \mathrm{rpm}$, unity hub flow coefficient), the diameter of the last stage of the LPT should be twice that of the TVC. The mass flow rate was selected to be $50 \mathrm{~kg} / \mathrm{s}$ to ensure safe design at a hub-to-tip ratio equals to 0.5 . The novel TVC comprises an axial compressor rotor followed by an axial turbine rotor is proposed to reduce losses. A quasi 3D design was applied to ensure equal work for the compressor and turbine at all spanwise sections. For the low-pressure steam turbine running at $50 \mathrm{~kg} / \mathrm{s}$, an equal work per stage was assumed to find the most appropriate and economical number of stages. A threestage design was selected to satisfy an accelerating relative flow condition across the last stage at all spanwise sections and over a range of hub flow coefficients from 0.8 to 1.2 . While the total-to-static efficiency is higher at $\phi_{h}=0.8$ by $5.7 \%$, the size of LPT is larger by $13.3 \%$ compared with $\phi_{h}=1.2$, which means higher manufacturing costs lower flow coefficient designs.

\section{Acknowledgement}

Thanks to Allah, thanks to Prof. Amin for his efforts, and finally, thanks to Dr. Shady for his time and efforts.

\section{References}

[1] Khattak, Muhammad Adil, Mohammad Azfar Haziq Ayoub, Muhammad Ariff Fadhlillah Abdul Manaf, Mohd Faidhi Mahrul, Mohd Ridwan Mohd Juhari, Mira Idora Mustaffa, and Suhail Kazi. "Global energy security and European Union: A review." Journal of Advanced Research in Applied Sciences and Engineering Technology 11, no. 1 (2018): 64-81.

[2] Ab Ghani, Sharin, and Idris Mohd Yusoff. "Comparative Study of Residential Lighting Technologies." Journal of Advanced Research in Applied Sciences and Engineering Technology 14, no. 1 (2019): 8-20. 
[3] Kumar, P. Vishwanath, Ajay Kumar Kaviti, Om Prakash, and K. S. Reddy. "Optimization of design and operating parameters on the year round performance of a multi-stage evacuated solar desalination system using transient mathematical analysis." International Journal of Energy \& Environment 3, no. 3 (2012).

[4] Kalogirou, Soteris A. "Seawater desalination using renewable energy sources." Progress in energy and combustion science 31, no. 3 (2005): 242-281. https://doi.org/10.1016/j.pecs.2005.03.001

[5] Saidi, Syahrul Affandi, Syarifah Idrus Sofia Syed Mohd Yamin, Mohd Sharizan Md Sarip, and Wan Azani Mustafa. "Sensor Application for Soil Treatment and Behaviour." Journal of Advanced Research in Applied Sciences and Engineering Technology 13, no. 1 (2018): 8-15.

[6] Mobarak, A. "Techno-economic evaluation of a novel thermal cycle for electricity generation and fresh water production from solar ponds." Classified Report DRTPC Publication 221-86 (1986).

[7] Mobarak, Amin. "A novel combined low temperature cycle for electricity and fresh water production." Journal of Solar Energy Engineering 137, no. 1 (2015): 014501. https://doi.org/10.1115/1.4027930

[8] A. Mobarak, S. Ali and M. Shawky. "A Novel Turbo-Vapor Axial Compressor." Egypt/Cairo Patent 2020/798 (Submitted for Acceptance), 11 June 2020.

[9] Karim Nice. "How turbochargers work." HowStuffWorks, 2021.

[10] Wilkes, Jason, B. Pettinato, R. Kurz, J. Hollingsworth, D. Zhang, M. Taher, C. Kulhanek, F. Werdecker, D. Büche, and G. Talabisco. "Compression Machinery for Oil and Gas." (2019).

[11] Dick, Erik. "Fundamentals of Turbomachines, volume 109 of Fluid Mechanics and Its Applications." Springer Netherlands. doi 10 (2015): 978-94. https://doi.org/10.1007/978-94-017-9627-9

[12] Korpela, Seppo A. Principles of turbomachinery. John Wiley \& Sons, 2019.

[13] S.Mikhail, P. A. Mobarak, and M. G.Khalafallah. "Basics of Turbomachines." Cairo: Faculty of Engineering, Cairo University, 2003.

[14] Holmgren, M. "X Steam, Thermodynamic properties of water and steam MATLAB Central File Exchange." (2020).

[15] Aungier, R. H., and S. Farokhi. "Axial-Flow Compressors: a Strategy for Aerodynamic Design and Analysis." Appl. Mech. Rev. 57, no. 4 (2004): B22-B22. https://doi.org/10.1115/1.1786589

[16] Aungier, R. H. "Axial-flow and radial inflow turbine design and analysis." Turbine aerodynamics 2006 (2006). https://doi.org/10.1115/1.802418

[17] Munyoki, Dickson, Markus Schatz, and Damian M. Vogt. "Detailed numerical study of the main sources of loss and flow behavior in low pressure steam turbine exhaust hoods." In Turbo Expo: Power for Land, Sea, and Air, vol. 50954, p. V008T29A004. American Society of Mechanical Engineers, 2017. https://doi.org/10.1115/GT2017-63269 